\title{
Different responses to anti-programmed cell death protein 1 (PD-1) immunotherapy in a patient with Lynch syndrome and metachronous urothelial and colon cancer: A case report
}

\author{
YU FENG $^{1 *}$, YUFENG CAO $^{2 *}$, MINGMING YUAN $^{3}$, RONGRONG CHEN $^{3}, \mathrm{XUE} \mathrm{JI}^{1}$ and XINGSHENG HU ${ }^{1}$ \\ ${ }^{1}$ Department of Medical Oncology, National Cancer Center/National Clinical Research Center for Cancer/Cancer Hospital, \\ Chinese Academy of Medical Sciences and Peking Union Medical College, Beijing 100021; ${ }^{2}$ Department of Oncology, \\ The Second Affiliated Hospital of Shandong University of Traditional Chinese Medicine, Jinan, Shandong 250001; \\ ${ }^{3}$ Department of R\&D, Geneplus-Beijing Institute, Beijing 102206, P.R. China
}

Received November 16, 2018; Accepted June 21, 2019

DOI: 10.3892/ol.2019.10909

\begin{abstract}
Lynch syndrome (LS), as a result of the germline mutations in DNA mismatch repair genes, is characterized by the increased risk of endometrium, colon, and urinary tract cancer. Individuals with this disorder may occasionally have multiple primary carcinomas. Regardless of tumor type, pembrolizumab was approved for the treatment of patients with unresectable or metastatic mismatch repair deficient tumors, which may be an optional therapeutic method for patients with LS with multiple primary carcinomas. This case study is of a MSH2-deficient patient with LS with metachronous urothelial and colon cancer, who received pembrolizumab treatment for 8 months. The responses of the two primary sites to immunotherapy differed. Based on the changes of tumor markers and tumor size illustrated by imageological examinations, no response was observed in the sigmoid colon lesion, whereas an immune-associated phenomenon known as pseudoprogression was detected in the ureteral lesion. Immunotherapy was innovatively applied to the patient with multiple primary carcinomas. This case proposes a novel concept in which immunotherapy may potentially control the cancer growth in patients with LS and multiple primary carcinomas. However, further large-scale investigations are required. Furthermore, it raises a challenge to monitor the effectiveness of immunotherapy.
\end{abstract}

Correspondence to: Professor Xingsheng Hu, Department of Medical Oncology, National Cancer Center/National Clinical Research Center for Cancer/Cancer Hospital, Chinese Academy of Medical Sciences and Peking Union Medical College, 17 Panjiayuannanli Road, Chaoyang, Beijing 100021, P.R. China E-mail: huxingsheng66@163.com

*Contributed equally

Key words: Lynch syndrome, metachronous urothelial and colon cancer, pembrolizumab, pseudoprogression

\section{Introduction}

Lynch syndrome (LS), also called hereditary nonpolyposis colorectal cancer (HNPCC), is a hereditary cancer predisposition syndrome that causes an increased risk for many types of cancers, including colorectal cancer and cancers of the endometrium, stomach, ovary, small bowel, as well as the urinary tract (1). The definitive diagnosis of LS is based on the identification of the germline pathogenic variants of mismatch repair (MMR) genes, mainly MLH1, PMS2, MSH2 and MSH6 (1). Defects in MMR proteins commonly result in the accumulation of genetic errors during DNA replication and therefore lead to high microsatellite instability (MSI-H) (1). Pembrolizumab, an anti-programmed cell death 1 (PD-1) antibody, shows promising efficacy for MSI-H or deficient MMR (dMMR) tumors $(2,3)$. Through the pooled analysis of 5 single-arm clinical studies (KEYNOTE $016,164,012,028,158)$, the objective response rate (ORR) was $36 \%$ in MSI-H or dMMR patients with colorectal cancer (CRC) and $46 \%$ in 14 non-CRC cancers, which promoted the first tissue/site agnostic approval of pembrolizumab in unresectable or metastatic, MSI-H or dMMR solid tumors (4). The results obtained in the current study suggested that different cancers with MSI-H or dMMR have different responses to anti-PD-1 therapy. However, the underlying mechanism is unknown.

Synchronous or metachronous multiple primary carcinomas are found in a subset of patients with LS. It has been previously reported that $>60 \%$ of patients with LS with rectal cancer develop colon cancer within 30 years (5). The 10-year cumulative risk of endometrial cancer for patients with LS firstly diagnosed with CRC was $23 \%$ (6). Despite this, to the best of our knowledge, there is a lack of a well-established treatment mode, therefore, this case aimed to investigate the therapeutic choice for the rare concurrent urothelial and colon cancers. We reported the response of a patient with LS with metachronous urothelial and colon cancers to pembrolizumab treatment.

\section{Case report}

A 38-year-old male presented to the Cancer Hospital, Chinese Academy of Medical Sciences (Beijing, China), complaining 
of progressive symptoms, including frequent and urgent micturition, and increased nocturia without apparent causes since February 2015. His maternal grandmother was diagnosed with carcinoma of the rectum at 39 years old. His mother was diagnosed with endometrial cancer at 39 and a metachronous adenocarcinoma of colon at 59 years old. His three maternal uncles were diagnosed with liver cancer. In December 2015, computed tomography (CT) imaging and magnetic resonance imaging (MRI) revealed the left ureteral mass $(2.7 \times 2.9 \mathrm{~cm})$ and multiple enlarged lymph nodes. Paraaortic lymph nodes biopsy and histological examination confirmed the lesions to be urothelial carcinoma, and the tumor stage was evaluated as $\mathrm{T}_{4} \mathrm{~N}_{2} \mathrm{M}_{\mathrm{X}}$ with suspected lung metastasis according to the 2004 World Health Organization classification (7). The patient received 4 cycles of gemcitabine and cisplatin chemotherapy between January 2016 and March 2016. CT scans in July 2016 suggested that the tumor became larger $(4.5 \times 3.8 \mathrm{~cm})$. Therefore, the patient was treated with paclitaxel liposome combined with tegafur, gimeracil, and oteracil potassium capsules between July 2016 and August 2016. In September 2016, the patient underwent regular chest and abdomen/pelvis CT scans, and the results showed local thickening of sigmoid colon wall (the thickest part was $\sim 1.6 \mathrm{~cm}$, and $\sim 6.7 \mathrm{~cm}$ in length) and multiple enlarged lymph nodes on the outside of the intestinal wall. Further colonoscopy revealed an ulcerative tumor on sigmoid colon. The hematoxylin and eosin (H\&E)-stained slides were reviewed by pathologists to confirm the diagnosis of moderately differentiated adenocarcinoma (Fig. 1A). Briefly, tissue samples were placed in $10 \%$ formalin for $24 \mathrm{~h}$ at room temperature and embedded in paraffin. Immunohistochemical staining (IHC) was performed using automated standard procedures (EnVision $^{\mathrm{TM}}$ Flex+ Detection System; cat. no. K8002; Dako; Agilent Technologies, Inc.). The standardized protocol supplied by the manufacturer was followed. The following primary antibodies were used: mutL homolog 1 (MLH1; cat. no. MAB-0642), mutS homolog 2 (MSH2; cat. no. MAB-0291), mutS homolog 6 (MSH6; cat. no. MAB-0643), PMS1 homolog 2, mismatch repair system component (PMS2; cat. no. MAB-0656), PD-1 (cat. no. MAB-0734), cytokeratin 20 (CK20; cat. no. Kit-0025), CD7 (cat. no. Kit-0021) and IL-12 P40 monomer (P40; cat. no. RMA-0815; all from Fuzhou Maixin Biotech Co., Ltd.), HER-2 (cat. no. 790-4493), BRAF-V600E (cat. no. 790-4855) and PD-L1 (cat. no. 741-4905; all from Roche Diagnostics) and caudal type homeobox 2 (CDX2; cat. no.ZA-0520; OriGene Technologies, Inc.). All antibodies were ready-to-use and were used without dilution. The IHC staining demonstrated that the tumor was positive for HER-2 (+), CDX2) (3+) and CK20 (3+), and was negative for CD7 and P40, which is significantly different from that of metastatic lymph nodes of urothelial carcinoma (HER-2: ++; fluorescence in situ hybridization revealed no amplification; CDX2: -; CK20: ++; CD7: +++; P40: +++). In addition, BRAF-V600E, PD-1 and PD-L1 for colon were not expressed. For MMR proteins, MSH2 was negative, however this was not the case for MLH1, MSH6 and PMS2 (Fig. 1). A diagnosis of primary moderately differentiated sigmoid adenocarcinoma was made.

Taking into consideration the clinical diagnosis, the MSH2-deficiency and the overwhelming family history, the patient was suspected to harbor a germline mutation in MMR genes, especially in MSH2. A genetic test was performed using DNA from the patient's peripheral blood by next-generation sequencing (Geneplus-Beijing Institute). As a result, 43 somatic mutations were identified and a germline variant was detected on $M S H 2$ (Table SI; Fig. 2A). The $M S H 2$ c. $1759+1 \mathrm{G}>\mathrm{T}$ germline variant is a novel splice site mutation that may affect splicing process and result in a defective MSH2 protein $(8,9)$. According to the American College of Medical Genetics and Genomics guidelines (10), the variant was judged as a likely pathogenic mutation. Sanger sequencing was performed to confirm the presence of $M S H 2$ c. $1759+1 \mathrm{G}>\mathrm{T}$ variant as previously described (11) (Fig. 2B). Therefore, the patient was finally diagnosed as MSH2-associated LS with urothelial and colon cancer. As the sigmoid colon lesions were observed 6 months after the diagnosis of urothelial carcinoma, it should be defined as metachronous carcinoma.

After 3 cycles of chemotherapy (irinotecan and pemetrexed) combined with bevacizumab treatment, the tumors progressed. The patient was subsequently administered pembrolizumab treatment (200 mg every three weeks) in December 2016. Compared with the lesions before pembrolizumab treatment (Fig. 3A and D), the imaging examinations in March 2017 suggested that the sigmoid colon lesion slightly increased and the ureteral lesion had progressed (Fig. 3B and E). In view of the notable level reduction of tumor markers detected by electrogenerated chemiluminescence (Table I), the patient was suspected to have pseudoprogression and therefore continued pembrolizumab treatment for another 4 months. The PET-CT performed in July 2017 showed the shrinkage of the left ureteral tumor. By contrast, progression was present on the sigmoid colon wall (Fig. 3C and F). According to the Response Evaluation Criteria In Solid Tumors v1.1 (12), the sigmoid colon lesion was evaluated as stable disease (SD) at the first evaluation, and progressive disease (PD) at the second evaluation, while the ureteral lesion was assessed as PD at the first evaluation and partial response (PR) at the second evaluation. Taking into consideration the unsatisfactory efficacy, the patient then returned to his local hospital to receive symptomatic and supportive treatment.

\section{Discussion}

LS is an autosomal-dominant disease caused by a pathogenic germline mutation in a DNA MMR, including $M L H 1, M S H 2$, MSH6, PMS2, or EPCAM gene $(13,14)$. Individuals with LS may have increased risk for many types of cancer. Particularly, the lifetime risk of colorectal cancer and endometrial cancer are 42 and $35 \%$, respectively $(13,14)$. LS is also associated with an increased risk of gastric cancer, ovarian cancer, hepatobiliary tract cancer, urinary tract cancer, small bowel cancer, brain cancer, pancreatic cancer, as well as sebaceous neoplasms $(13,14) . M S H 2$, being the most frequently mutated gene, has a cumulative incidence of ureter and kidney cancers for patients with MSH2-associated LS of $17.8 \%$ by 75 years old (15). LS-associated hepatocellular carcinoma, although not common, was reported in a number of cases $(16,17)$. In this case, a germline $\mathrm{MSH} 2$ mutation was identified in a patient with metachronous urothelial and colon cancer. The cancerous manifestations of the patient and his family members were all 


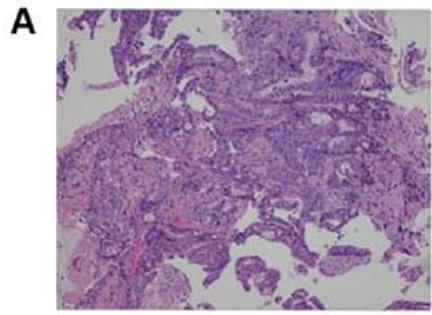

H\&E

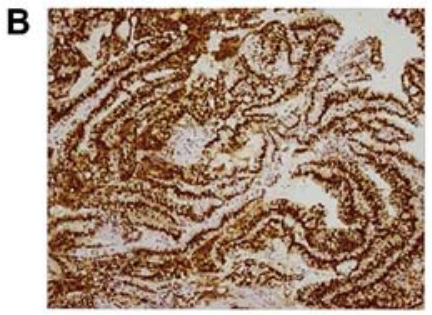

$$
\text { MLH1 }
$$

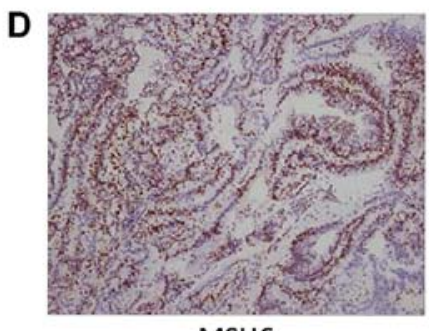

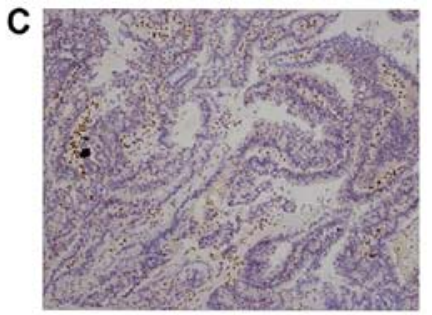

MSH2

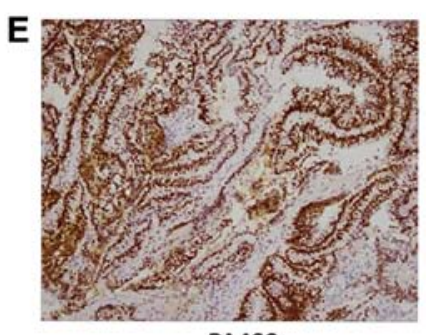

PMS2

Figure 1.H\&E and immunohistchemical staining of the sigmoid colon lesion. (A) H\&E and immunohistochemical staining of (B) MLH1, (C) MSH2, (D) MSH6 and (E) PMS2 of the sigmoid colon lesion. MLH1, MutL homolog 1; PMS2, PMS1 homolog 2, mismatch repair system conponent; MSH6, mutS homolog 6; MSH2, MutS homolog 2. Magnification, x100.

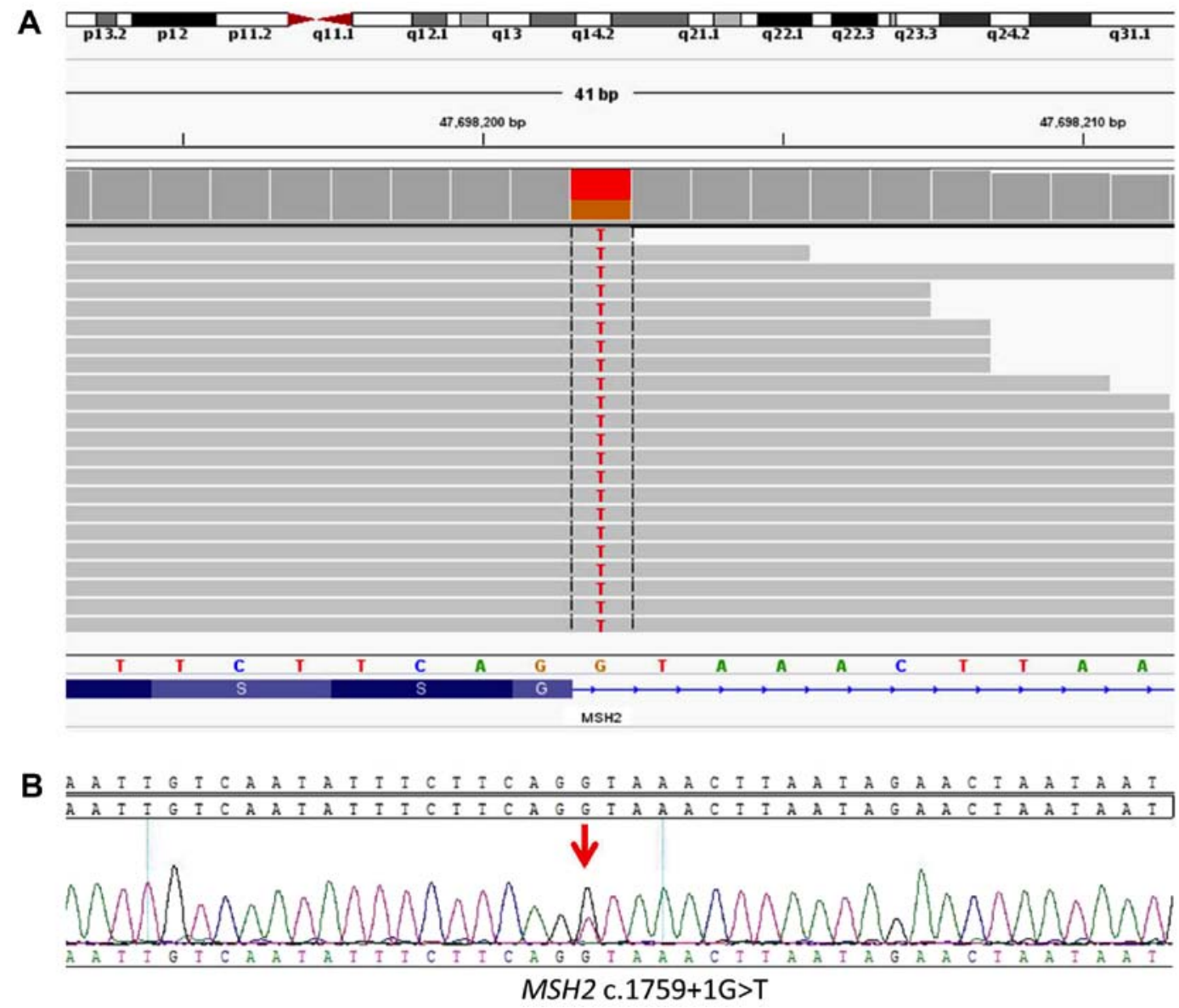

Figure 2. $M S H 2$ germline mutation identified by NGS and confirmed by Sanger sequencing. (A) The reads map of $M S H 2$ c.1759+1G $>$ T germline mutation detected by NGS. (B) Sanger sequencing of the $M S H 2$ c.1759+1G $>$ T germline mutation for this patient, indicated by the red arrow. MSH2, MutS homolog 2; NGS, next-generation sequencing.

considered as part of the tumor spectrum of LS, though this case was unable to get access to other family members' DNA samples.
The traditional two-hit model is widely used to describe the genesis of LS. A germline loss-of-function mutation accompanied with somatic inactivation of the other allele 
Table I. Tumor markers of the patient between November 2016 and July 2017 as detected by electrogenerated chemiluminescence.

\begin{tabular}{|c|c|c|c|c|}
\hline Markers (unit) & $11 / 28 / 2016$ & $03 / 20 / 2017$ & $07 / 05 / 2017$ & Upper limit of the normal range \\
\hline CA19-9 (U/ml) & 307.9 & 133.3 & 116.7 & 37 \\
\hline CA72-4 (U/ml) & 82.04 & 41.84 & 56.48 & 9.8 \\
\hline CEA (ng/ml) & 216.2 & 159.5 & 327 & 5 \\
\hline
\end{tabular}

A

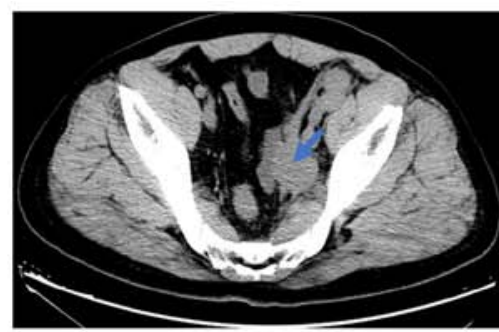

D

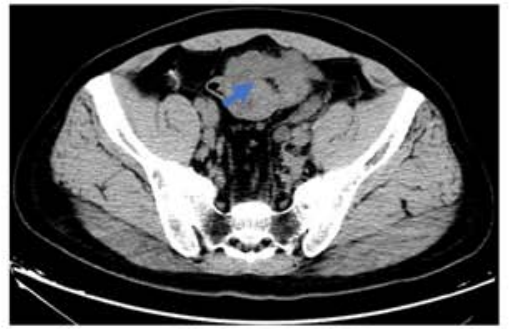

B

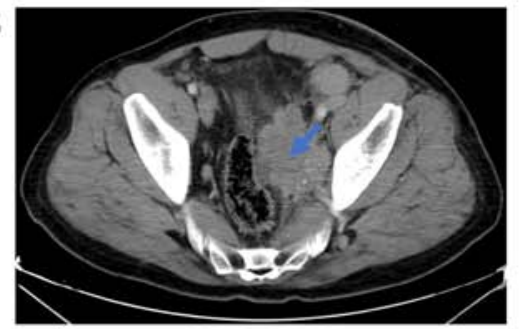

E

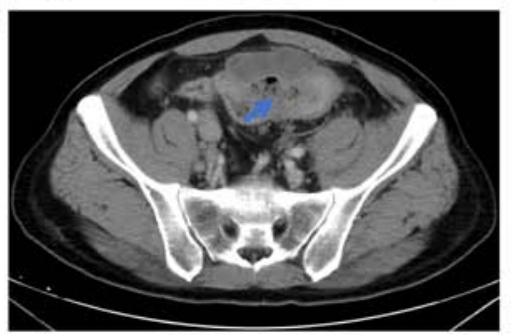

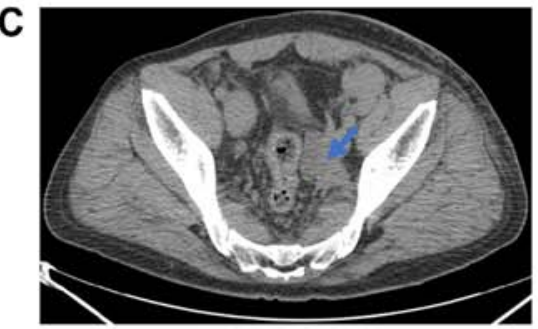

$\mathbf{F}$

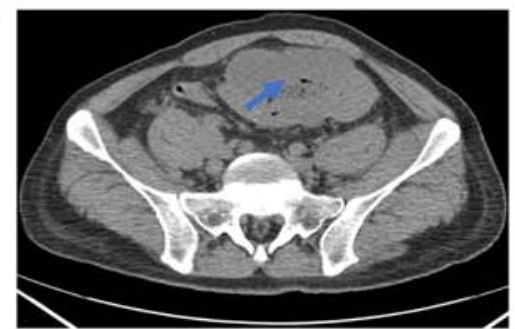

Figure 3. Image examinations demonstrate the changes of left ureteral and colon tumors during the period of pembrolizumab treatment. The arrow marks the location of the tumors. (A) represents the changes of left ureteral tumors before pembrolizumab treatment, (B) at 4 months after treatment and (C) at 8 months after treatment. (D) represents the changes of colon tumors before pembrolizumab treatment, (E) at 4 months after treatment and (F) at 8 months after treatment. The colon wall increased in thickness steadily, while the ureteral lesion increased in size at 4 months, and markedly decreased at 8 months.

promotes tumorigenesis (18). In this case, the patient carried a germline $\mathrm{MSH} 2$ mutation, while no $\mathrm{MSH} 2$ somatic mutation was detected. Biallelic inactivation of $M S H 2$ may be caused by other mechanisms, such as loss of heterozygosity (19). IHC revealed the loss of expression of MSH2 protein and made the conclusion of dMMR.

Given that MMR deficiency is a validated biomarker for immunotherapy $(2,20)$, the patient switched to pembrolizumab treatment after failure of chemotherapy. The lesion on the sigmoid colon appeared to have no response to pembrolizumab with continuous aggravation, whereas the ureteral lesion was evaluated as PD at the first response evaluation, and as PR at the second evaluation, which was considered as pseudoprogression.

Pseudoprogression is an immunotherapy-associated phenomenon. Increased tumor size, revealed by imaging examination, may reflect tumor cells that are infiltrated with lymphocytes and macrophages (21). These patients may benefit from immunotherapy, however may switch to other treatment regimens based on the imaging evaluation. These inflammatory-based processes have prompted the development of immune-related response criteria (irRC), which can identify $10 \%$ of patients with pseudoprogression (22). However, recent studies demonstrated that circulating tumor DNA monitoring can accurately distinguish pseudoprogression from true progression in patients with lung adenocarcinoma and melanoma treated with immunotherapy $(23,24)$.
The response to PD-1 inhibition varies among different tumor types (25). The efficacy of pembrolizumab in patients with previously treated urothelial carcinoma was evaluated in study KEYNOTE-045. For patients treated with pembrolizumab, ORR was $21 \%$ and overall survival time was 10.3 months (26). A phase 2 clinical study evaluated the efficacy of pembrolizumab in metastatic carcinomas with or without dMMR (19). ORR for dMMR colorectal cancers was $40 \%$. For MMR proficient colorectal cancers, ORR was 0 (19). The objective response rate of pembrolizumab in MSI-H or dMMR non-CRC cancers $(36 \%)$ is higher than in patients with CRC $(46 \%)(4,25)$. In this case, no response was observed on sigmoid colon lesion, while the ureteral lesion achieved PR after pseudoprogression Immunotherapy-associated biomarkers may contribute to the different responses. IHC analysis of colon lesion showed negative expression of MSH2 and PD-L1. However, the expression of these proteins in ureteral lesion was unknown and no tumor tissue was left to allow the performance of PD-1/PD-L1 IHC staining. One research investigated the states of MSI, PD-L1 and TMB in 11,348 patients with cancer (27). High MSI, PD-L1 and TMB were identified in 5.7, 6.7 and $7.2 \%$ of colorectal adenocarcinoma (1,395 patients), and in $0.0,16.8$ and $42.7 \%$ of bladder cancer (143 patients) (27). Compared with bladder cancer, the percentage of MSI was higher in colorectal carcinoma. On the contrary, TMB-H and PD-L1 positivity were present 
more often in bladder cancer, which may explain the different responses of the two primary sites (27).

This case presents the treatment course in a challenging case of a patient with MSH2-associated LS manifested with metachronous ureteral urothelial cancer and colon adenocarcinoma. Taking into consideration dMMR confirmed by IHC staining, the patient was treated with immune checkpoint inhibitor pembrolizumab, after disease progression on chemotherapy. The response patterns of the two primary lesions to pembrolizumab differed. This case report discussed the potential explanations underlying this phenomenon; however, further clinical investigations are required.

\section{Acknowledgements}

Not applicable.

\section{Funding}

No funding was received.

\section{Availability of data and materials}

The datasets used and/or analyzed during the current study are available from the corresponding author on reasonable request.

\section{Authors' contributions}

YF, YC and $\mathrm{XH}$ were major contributors in writing the manuscript. YF and $\mathrm{XH}$ were responsible for the analysis of clinical information. MY and RC were responsible for genetic analysis and literature review. MY edited the manuscript. YC and XJ conducted imaging examinations and analysis. All authors have read and approved the final version of the manuscript.

\section{Ethics approval and consent to participate}

Not applicable.

\section{Patient consent for publication}

Written informed consent was obtained from the patient for publication of the clinical data and any accompanying images.

\section{Competing interests}

The authors declare no conflicts of interest.

\section{References}

1. Lynch HT, Snyder CL, Shaw TG, Heinen CD and Hitchins MP Milestones of Lynch syndrome: 1895-2015. Nat Rev Cancer 15: 181-194, 2015.

2. Le DT, Durham JN, Smith KN, Wang H, Bartlett BR, Aulakh LK, Lu S, Kemberling H, Wilt C, Luber BS, et al: Mismatch repair deficiency predicts response of solid tumors to PD-1 blockade. Science 357: 409-413, 2017.

3. Marcus L, Lemery SJ, Keegan P and Pazdur R: FDA approval summary: Pembrolizumab for the treatment of microsatellite instability-high solid tumors. Clin Cancer Res 25: 3753-3758, 2019.
4. Food and Drug Administration (FDA): FDA grants accelerated approval to pembrolizumab for first tissue/site agnostic indication. FDA, Silver Spring, MD, 2018. https://www.fda. gov/Drugs/InformationOnDrugs/ApprovedDrugs/ucm560040. htm. Accessed July 30, 2018.

5. Win AK, Parry S, Parry B, Kalady MF, Macrae FA, Ahnen DJ, Young GP, Lipton L, Winship I, Boussioutas A, et al: Risk of metachronous colon cancer following surgery for rectal cancer in mismatch repair gene mutation carriers. Ann Surg Oncol 20: 1829-1836, 2013.

6. Obermair A, Youlden DR, Young JP, Lindor NM, Baron JA, Newcomb P, Parry S, Hopper JL, Haile R, Jenkins MA, et al: Risk of endometrial cancer for women diagnosed with HNPCC-related colorectal carcinoma. Int J Cancer 127: 2678-2684, 2010.

7. Eble JL, Sauter G and Epstein JI: Pathology and genetics of tumours of the urinary system and male genital organs: WHO classification of tumours. World Health Organ, 2004.

8. Bianchi F, Rosati S, Belvederesi L, Loretelli C, Catalani R, Mandolesi A, Bracci R, Bearzi I, Porfiri E and Cellerino R: MSH2 splice site mutation and endometrial cancer. Int J Gynecol Cancer 16: 1419-1423, 2006.

9. Rakobradović J, Krivokuća A, Jovandić S, Kesić V and Branković-Magić M: Confirmation of damaging effect of MSH2 c. $2634+1 \mathrm{G}>\mathrm{C}$ mutation on splicing, its classification and implications for counseling. Cancer Genet 239: 1-7, 2019.

10. Richards S, Aziz N, Bale S, Bick D, Das S, Gastier-Foster J, Grody WW, Hegde M, Lyon E, Spector E, et al: Standards and guidelines for the interpretation of sequence variants: A joint consensus recommendation of the American College of Medical Genetics and Genomics and the Association for Molecular Pathology. Genet Med 17: 405-424, 2015.

11. Liu Q, Wang LA, Su J, Tong D, Lan W, Wang L, Liu G, Zhang J, Zhang VW, Zhang D, et al: Giant bilateral adrenal myelolipomas in two Chinese families with congenital adrenal hyperplasia. Endocr Connect: Sep 1, 2018 (Epub ahead of print).

12. Eisenhauer EA, Therasse P, Bogaerts J, Schwartz LH, Sargent D, Ford R, Dancey J, Arbuck S, Gwyther S, Mooney M, et al: New response evaluation criteria in solid tumours: revised RECIST guideline (version 1.1). Eur J Cancer 45: 228-247, 2009.

13. Bonadona V, Bonaiti B, Olschwang S, Grandjouan S, Huiart L, Longy M, Guimbaud R, Buecher B, Bignon YJ, Caron O, et al: Cancer risks associated with germline mutations in MLH1, MSH2 and MSH6 genes in Lynch syndrome. JAMA 305: 2304-2310, 2011.

14. Møller P, Seppälä T, Bernstein I, Holinski-Feder E, Sala P, Evans DG, Lindblom A, Macrae F, Blanco I, Sijmons R, et al: Cancer incidence and survival in Lynch syndrome patients receiving colonoscopic and gynaecological surveillance: First report from the prospective Lynch syndrome database. Gut 66: 464-472, 2017.

15. Møller P,Seppälä TT, Bernstein I,Holinski-Feder E, Sala P, Gareth Evans D, Lindblom A, Macrae F, Blanco I, Sijmons RH, et al: Cancer risk and survival in path_MMR carriers by gene and gender up to 75 years of age: A report from the Prospective Lynch Syndrome Database. Gut 67: 1306-1316, 2018.

16. Casper M, Weber SN, Kloor M, Müllenbach R, Grobholz R, Lammert F and Zimmer V: Hepatocellular carcinoma as extracolonic manifestation of Lynch syndrome indicates SEC63 as potential target gene in hepatocarcinogenesis. Scand J Gastroenterol 48: 344-351, 2013.

17. Vernez M, Hutter P, Monnerat C, Halkic N, Gugerli O and Bouzourene H: A case of Muir-Torre syndrome associated with mucinous hepatic cholangiocarcinoma and a novel germline mutation of the MSH2 gene. Fam Cancer 6: 141-145, 2007.

18. Yan H, Jin H, Xue G, Mei Q, Ding F, Hao L and Sun SH: Germline hMSH2 promoter mutation in a Chinese HNPCC kindred: Evidence for dual role of LOH. Clin Genet 72: 556-561, 2007.

19. Hemminki A, Peltomäki P, Mecklin JP, Järvinen H, Salovaara R, Nyström-Lahti M, de la Chapelle A and Aaltonen LA: Loss of the wild type MLH1 gene is a feature of hereditary nonpolyposis colorectal cancer. Nat Genet 8: 405-410, 1994.

20. Le DT, Uram JN, Wang H, Bartlett BR, Kemberling $H$, Eyring AD, Skora AD, Luber BS, Azad NS, Laheru D, et al: PD-1 blockade in tumors with mismatch-repair deficiency. N Engl J Med 372: 2509-2520, 2015.

21. Wang Q, Gao J and Wu X: Pseudoprogression and hyperprogression after checkpoint blockade. Int Immunopharmacol 58: 125-135, 2018.

22. Agarwala SS: Practical approaches to immunotherapy in the clinic. Semin Oncol 42 (Suppl 3): S20-S27, 2015. 
23. Guibert N, Mazieres J, Delaunay M, Casanova A, Farella M Keller L, Favre G and Pradines A: Monitoring of KRAS-mutated ctDNA to discriminate pseudo-progression from true progression during anti-PD-1 treatment of lung adenocarcinoma. Oncotarget 8: 38056-38060, 2017.

24. Lee JH, Long GV, Menzies AM, Lo S, Guminski A Whitbourne K, Peranec M, Scolyer R, Kefford RF, Rizos H and Carlino MS: Association between circulating tumor DNA and pseudoprogression in patients with metastatic melanoma treated with anti-programmed cell death 1 antibodies. JAMA Oncol 4: 717-721, 2018.

25. Yarchoan M, Hopkins A and Jaffee EM: Tumor mutational burden and response rate to PD-1 inhibition. N Engl J Med 377: 2500-2501, 2017.
26. Bellmunt J, de Wit R, Vaughn DJ, Fradet Y, Lee JL, Fong L, Vogelzang NJ, Climent MA, Petrylak DP, Choueiri TK, et al: Pembrolizumab as second-line therapy for advanced Urothelial Carcinoma. N Engl J Med 376: 1015-1026, 2017.

27. Vanderwalde A, Spetzler D, Xiao N, Gatalica Z and Marshall J: Microsatellite instability status determined by next-generation sequencing and compared with PD-L1 and tumor mutational burden in 11,348 patients. Cancer Med 7: 746-756, 2018

(i) (9) This work is licensed under a Creative Commons Attribution-NonCommercial-NoDerivatives 4.0 International (CC BY-NC-ND 4.0) License. 